NASA Contractor Report 195373

AIAA-94-2983

\title{
The Use of Spontaneous Raman Scattering for Hydrogen Leak Detection
}

Wim A. de Groot

NYMA, Inc.

Brook Park, Ohio

August 1994

Prepared for

Lewis Research Center

Under Contract NAS3-25266

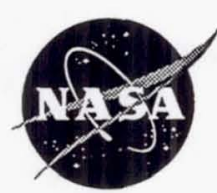

National Aeronautics and

Space Administration 


\title{
THE USE OF SPONTANEOUS RAMAN SCATTERING FOR HYDROGEN LEAK DETECTION
}

\author{
Wim A. de Groot ${ }^{\star}$ \\ NYMA Inc., \\ NASA Lewis Research Center \\ Cleveland, Ohio
}

\begin{abstract}
$\underline{\text { Abstract }}$
A fiber optic probe has been built and demonstrated that utilizes back scattered spontaneous Raman spectroscopy to detect and identify gaseous species. The small probe, coupled to the laser and data acquisition equipment with optical fibers, has applications in gaseous leak detection and process monitoring. The probe design and data acquisition system are described. Raman scattering theory has been reviewed and the results of intensity calculations of hydrogen and nitrogen Raman scattering are given. Because the device is in its developmental stage, only preliminary experimental results are presented here. Intensity scans across the rotationalvibrational Raman lines of nitrogen and hydrogen are presented. Nitrogen at a partial pressure of $0.077 \mathrm{MPa}$ was detected. Hydrogen at a partial pressure of $2 \mathrm{kPa}$ approached the lower limit of detectability with the present apparatus. Potential instrument improvements that would allow more sensitive and rapid hydrogen detection are identified.
\end{abstract}

\section{Introduction}

Propellant leakage is a significant issue for all launch systems that use cryogenic liquid propellants. Propellant systems that use liquid hydrogen experience leaks more frequently than those that use other, less volatile, propellants. This is due to the volatile nature of hydrogen which vaporizes readily and which makes it extremely difficult to contain. Furthermore, hydrogen leaks in air form a highly explosive mixture and therefore require immediate attention. This makes each hydrogen leak a matter of the highest concern. The Space Shuttle utilizes liquid hydrogen in its main propulsion system. As of December 1993, a total of 59 space shuttle launches had taken place. 49 of these flights were delayed or rescheduled adding up to a total of 120 individual delays with a total duration of 1800 delay days. ${ }^{1}$ Of these delay

\footnotetext{
${ }^{*}$ Sr. Research Engineer, Member AIAA
}

days, 165 were due to hydrogen leaks or hydrogen leak sensor malfunctions.

Leaks from the 20 gaseous and liquid STS fluid systems and associated servicing and ground support fluid systems are detected using 11 different detection methods. ${ }^{1}$ These different leak detection systems introduce considerable complexity and are extremely labor intensive. Many of these methods do not fully satisfy the criteria of leak detection, identification, and isolation (location), but are the best currently available.

Improvements in the leak resistance of fluid systems continues to be emphasized, however, leak detection will always be a necessary part of any fluid system. It may be possible to find an alternative leak detection scheme based on a mature technology that can offer improvements in leak detection capability of these systems. The only technology that currently has the maturity to satisfy most, if not all of the criteria for in-situ, realtime leak detection is spontaneous Raman scattering. In addition, a leak detection system based on spontaneous Raman scattering could be made small and light weight, so that it can be used during the preparation, in-flight, and return phases of the mission and, additionally, should not require intensive personnel impact.

Hydrogen leak detection using Raman spectroscopy is chosen for this investigation for previously mentioned reasons. Raman scattering from nitrogen is also investigated. Several researchers have pursued hydrogen leak detection based on Raman scattering. Adler-Golden et al. ${ }^{2}$ designed a system based on spontaneous Raman spectroscopy. The gas to be diagnosed needed to be collected and passed through a gas cell, precluding in-situ measurements. The measurement device weighed $16 \mathrm{~kg}$ and occupied a volume $0.02 \mathrm{~m}^{2}$. Caputo ${ }^{3}$ build a Raman Lidar (LIght Detection And Ranging) system on the back of a truck and performed tests at the Stennis Space Center (SSC) facility. 
The purpose of this paper is to describe a small light weight system based on Raman scattering which could be used in-flight and/or on the ground. The use of optical fibers with a depth of field makes it possible to do in-situ detection, identification, and location in real-time. This inexpensive and compact system can be expanded to accommodate multiple gases and/or liquids and can be easily updated with newly developed instrumentation especially in the area of optoelectronics.

\section{Theory}

Different types of molecules possess different internal molecular structure. Molecules of the same species but different phase (gas, liquid, or solid) also show differences in internal structure. Raman scattering relies on a laser beam to probe internal rotational and vibrational molecular structure. As a result of this laser excitation, each type of molecule emits a different scattering signature. Many gases and liquids can be identified by this signature.

A general overview of spontaneous Raman scattering is given in Appendix A. A more detailed description with in-depth theory can be found in Reference 4. Shirley ${ }^{5}$ investigates the use of spontaneous Raman scattering for temperature measurement on hydrogen inside the Space Shuttle fuel pre-burner and calculates the line strengths of individual rotational vibrational transitions between 600 and $1200 \mathrm{~K}$. Results from this study are used for predicting both the hydrogen and nitrogen spectra and signal intensities at ambient conditions. The data required to calculate the line strengths are found in Shirley's report and in Refs. 6 and 7. For completeness, they are listed in Table 1. A detailed description of these parameters is given in Appendix A. (For clarity it should be noted that the quantities $\gamma^{i}$ and $\beta_{e}{ }^{\prime}$ are both used in Shirley's report. It is the belief of the author that these quantities are the same, they both represent the derived polarizability anisotropy. This simultaneous use is confusing because $\gamma^{\prime}$ is the commonly used term whereas Refs. 6 and 8 use $\beta_{\mathrm{e}}$.)

The Raman spectra of hydrogen and nitrogen based on Raman scattering theory and the line intensities as presented by Shirley are calculated for $298 \mathrm{~K}$ and $0.1 \mathrm{MPa}$ pressure. The calculated Raman spectrum of hydrogen is shown in Figure 1. The excitation wavelength is $514.5 \mathrm{~nm}$, the green line of an Argon Ion laser. The pure rotational transitions are stronger than the rotationalvibrational transitions. They are very close to the laser line however, and difficult to optically separate from that line in real experimental situations. Therefore, the Q-branch of the rotational-vibrational transition, which are the next strongest transitions, are considered in this investigation. For hydrogen under excitation of the $514.5 \mathrm{~nm}$ line, the Q-branch is centered around $654.6 \mathrm{~nm}$. The O- and S-branches are calculated to be an order of magnitude weaker, and are further apart spectrally. They are almost imperceptible in Figure 1.

\begin{tabular}{|l|l|l|l|c|}
\hline Param. & $\mathrm{H}_{2}$ & Ref. & $\mathrm{N}_{2}$ & Ref. \\
\hline$\beta_{0}\left(\mathrm{~cm}^{3}\right)$ & $\begin{array}{l}0.314 \\
\times 10^{-24}\end{array}$ & 5 & $\begin{array}{l}0.696 \\
\times 10^{-24}\end{array}$ & 6 \\
\hline$\beta_{\mathrm{e}}\left(\mathrm{cm}^{3}\right)$ & $\begin{array}{l}0.288 \\
\times 10^{-24}\end{array}$ & 6 & $\begin{array}{l}0.691 \\
\times 10^{-24}\end{array}$ & 6 \\
\hline$\chi$ & 0.38 & 5 & 0.45 & 6 \\
\hline$\alpha^{6}\left(\mathrm{~cm}^{2}\right)$ & $\begin{array}{l}1.230 \\
\times 10^{-16}\end{array}$ & 5 & $\begin{array}{l}1.75 \\
\times 10^{-16}\end{array}$ & 7 \\
\hline$\gamma^{\prime}\left(\mathrm{cm}^{2}\right)$ & $\begin{array}{l}1.02 \\
\times 10^{-16}\end{array}$ & 5 & $\begin{array}{l}1.40 \\
\times 10^{-16}\end{array}$ & 6 \\
\hline$r_{\mathrm{e}}\left(\mathrm{cm}^{2}\right)$ & $\begin{array}{l}0.741 \\
\times 10^{-8}\end{array}$ & 5 & $\begin{array}{l}1.098 \\
\times 10^{-8}\end{array}$ & 6 \\
\hline
\end{tabular}

Table 1: Polarizability Matrix Element Data

Only vibrational Stokes scattering is considered. because at ambient temperatures only the vibrational ground state is populated. Furthermore, because the rotational $Q$-branch is several orders of magnitude stronger than the 0 and S- branches, we limited ourselves to measuring this Q-branch. The distribution of the line intensities of the Q-branch depends on the initial population. This in turn is governed by the temperature. At higher temperatures, higher rotational lines become populated and a broader distribution over more lines is expected, slightly complicating an accurate assessment of the total population, because the line intensity is proportional to the rotational quantum number. For an accurate number density measurement at higher temperatures therefore, the intensities of individual Q-branch lines have to be measured. In the current research where only ambient temperatures are considered, this dependency can be ignored.

The calculated Raman spectrum of nitrogen at ambient temperature is shown in Figure 2. Many more rotational lines are populated for nitrogen than for hydrogen, but because the rotational energy levels of nitrogen are much closer apart, the resulting line are closely spaced in the spectrum. All Q-branch lines are collapsing in a single line in this figure. A high resolution 
spectrometer or interferometer would be required to resolve the individual lines.

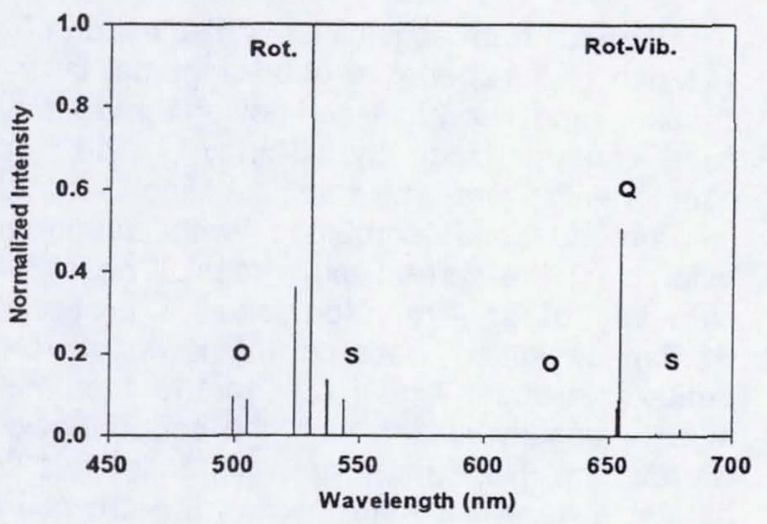

Fig. 1: Line intensities of the Raman spectrum of hydrogen at $298 \mathrm{~K}$

These calculations show that it is possible to use Raman scattering for gas detection and identification. As a first approximation, we assume that the temperature of leak gas to be detected does not vary significantly $\left(<20^{\circ} \mathrm{F}\right)$. This eliminates the temperature from consideration, and a direct measurement of the integrated scattering intensity of the lowest rotational lines gives the number density. A calculation of the Raman intensities at ambient temperature and with varying partial pressures verifies that for a constant temperature, the signal intensity is proportional to the number density.

Line broadening of the various Raman lines of hydrogen and nitrogen is a strong function of pressure and temperature. At ambient pressure and temperature, Doppler broadening dominates with a small contribution due to pressure broadening. For hydrogen at these conditions, additional line narrowing occurs due to Dicke line narrowing. ${ }^{9}$ Doppler broadening occurs when the molecule that scatters the photon simultaneously undergoes an elastic collision. ${ }^{5}$ Some of the recoil momentum imparted on the scattering molecule by the scattered photon might be absorbed by the colliding molecule. This causes the photon to have a slightly different Raman shift than without collision. The broadening depends on the frequency and weakly on the type of collisions and therefore on the pressure and on the collision partners. For total Raman Q-branch intensity measurements at ambient temperature and pressure, the broadening effects only vary with partial pressure of the different species, due to a change in type of collision partners. This implies that the contribution of broadening effects on the overall intensity measurement is negligible and that no special consideration has to be given to the effect of different line broadening phenomena. The intensity measured has a linear relationship with the hydrogen number density.

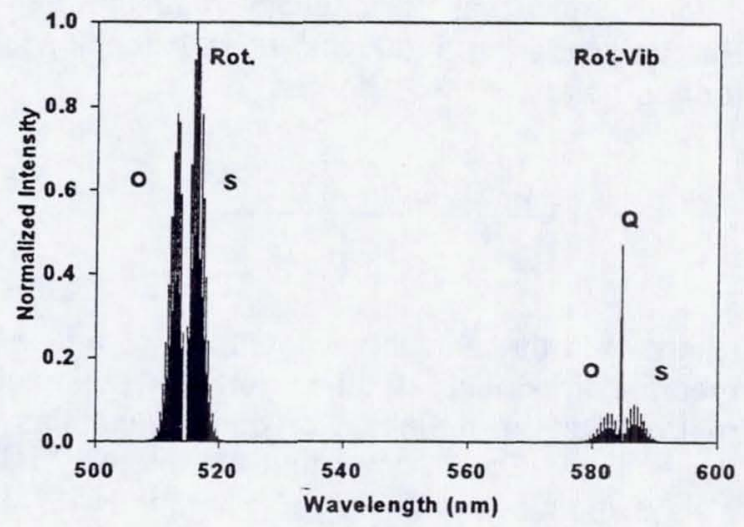

Fig. 2: Line intensities of the Raman spectrum of nitrogen at $298 \mathrm{~K}$

The Raman scattered light is very weak, despite the fact that all of the emitted scattering is distributed over a few lines. To detect such a signal, a sensitive detector is needed. A high quantum efficiency photo multiplier tube such as a Gallium-Arsenide tube with a quantum efficiency around $12 \%$ at $650 \mathrm{~nm}$, is the detector of choice. Despite the PM tube's sensitivity, the signal is still very weak, and for low number densities will be smaller then the noise associated with PMT tubes, such as shot noise and Johnson noise.

A lock-in amplifier scheme is utilized to extract this weak signal from the noise. In theory, a signal can be extracted from a signal with several orders of magnitude higher broadband noise. To accomplish lock-in detection, the excitation laser beam is chopped by means of an optical chopper. This generates a square wave temporal exposure of the gas volume, where the off-time contains noise only and the on-time contains the signal plus noise. The lock-in amplifier processes the photo multiplier signal into a DC signal with an amplitude proportional to the Raman signal amplitude. All noise except for those contributions with frequency very close or equal to the chopper frequency will be filtered out with a low pass filter. With a very good low pass filter, the measured signal is directly proportional to the scattering frequency signal with all noise removed. 


\section{Experimental}

Apparatus

Calculations based on Raman scattering theory ${ }^{10}$ show that the intensity ratio between the Raman scattering at $90^{\circ}$ and at $180^{\circ}$ (back scatter) for non-polarized excitation is:

$$
\frac{I_{90^{\circ}}}{I_{180^{\circ}}}=\frac{13 \frac{r^{r^{2}}}{\alpha^{\prime 2}}+45}{14 \frac{r^{r^{2}}}{\alpha^{\alpha^{2}}}+90}
$$

where $\gamma^{\prime}$ is the anisotropic component and $\alpha^{\prime}$ the mean component of the derived polarizability matrix. For the definition of these quantities, see Appendix A. Eq. 1 shows that the intensity ratio is always between $1 / 2$ for $\alpha^{\prime} \gg \gamma^{\prime}$ and $13 / 14$ for $\alpha^{i}<<\gamma^{\prime}$. Using the values from Table 1, Eq. 1 gives an intensity ratio for hydrogen of 0.541 and for nitrogen of 0.539 . This indicates a preference for $180^{\circ}$ angle observation. The confluence of incident and observed light paths gives a better spatial resolution for observation at $90^{\circ}$. Observation at $180^{\circ}$ causes the instrument to have a relatively long probe volume, which can be limited by masking the center of the observation optics. The stronger back scatter signal in combination with the requirement to measure with a finite depth of field, has led to the design of the test with back scatter collection optics.

An optical probe has been developed that combines laser beam delivery to the sample volume with the optical collection of Raman scattered light. Both the laser beam and the collected light are transmitted between the probe and the laboratory with optical fibers. A schematic of the optics head is shown in Figure 3. A laser beam, delivered by an optical fiber is collimated by means of a graded index (grin) lens appended to the fiber end. The available graded index lens has a diameter of $1.8 \mathrm{~mm}$, a pitch of 0.23 , and is designed for a wavelength of $1064 \mathrm{~nm}$. Adjusting the distance between the fiber and the lens provides an acceptable collimation $\left(0.12^{\circ}\right.$ divergence) of the laser light emerging from the fiber into a $1 \mathrm{~mm}$ diameter beam. This beam propagates through a dichroic mirror and is focused through a fast lens with a $F$-number $\left(F_{\#}\right)$ of 2.0 into a probe volume. The same lens collects the light scattered by the gas over a large solid angle. The light is reflected by the dichroic mirror and a fully reflective mirror and focused into a second fiber. The fully reflective mirror is masked off at the center. This improves spatial resolution and removes the laser light scattered from the dichroic optics and focusing lens without losing much of the solid collection angle.

The full data acquisition is displayed in Figure 4. Much of the theory to obtain optimal laser-fiber coupling and optimal laser power throughput has been summarized by Shirley. ${ }^{5}$ The optical components are selected based on these recommendations combined with requirements tailored for the current experiment. The $488.0 \mathrm{~nm}$ blue line of an Argon-Ion laser is preferred for Raman excitation, because the lower wavelength emits a stronger signal due to the fact that the signal intensity scales with the fourth power the wavelength. No Raman notch filter for this line is available, however, and the $514.5 \mathrm{~nm}$ green laser line of a 4 Watt laser has been selected.

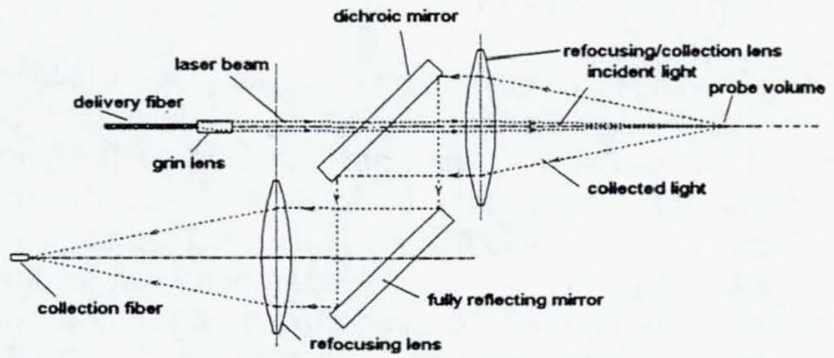

Fig 3: Raman gas detection diagnostics probe head

At the laser output, the beam is chopped with an optical chopper. The slotted wheel is equipped with six holes and is operated at a frequency of 300 to $500 \mathrm{~Hz}$. This creates a near square wave chopped laser beam that exposes the gas volume and allows the lock-in amplifier to process the signal. The chopper provides a TTL signal of the same frequency to the lock-in amplifier as a reference signal. A Faraday rotator decouples the laser from the fiber. This removes the contribution that the laser output coupler has on the effective reflectivity of the fiber ends. The critical power that can be coupled into the fiber is therefore increased, because this reduces the feedback from the ends of the fiber caused by Brillouin scattering, ${ }^{5}$

The laser beam is coupled through a microscope objective with a numerical aperture of 0.25 (N.A. $=\sin \{\alpha / 2\}$, where $\alpha$ is the acceptance angle of the fiber) into a $8 \mu \mathrm{m}$ diameter optical fiber. The microscope objective consists of optical components only without epoxy. This is required to prevent damage to the epoxy due to the high power level. Three available optical fibers were tested. A $4.3 \mu \mathrm{m}$ diameter fiber supported the 
transmission of only $30 \%$ of the laser power. This was due to the fact that the number of modes that were supported by the fiber was limited to only a few modes whereas the total laser power was spread over a larger number of modes. A $200 \mu \mathrm{m}$ diameter fiber supported the transmission of $85 \%$ of the total laser power but had to be discarded because the large fiber caused unacceptable divergence by the graded index lens in the probe head. The $8 \mu \mathrm{m}$ fiber that was selected supported sufficient modes to allow acceptable beam power transmission. Experimentally it was found that a 0.8 Watt laser beam was coupled and transmitted with a transmission efficiency of about $55 \%$, which delivered about 0.45 Watt power at the probe head. From these tests it was clear that there is a trade-off between power transmitted and acceptable collimation by the graded index lens. Additional tests should determine the maximum fiber diameter that still allows acceptable beam collimation.

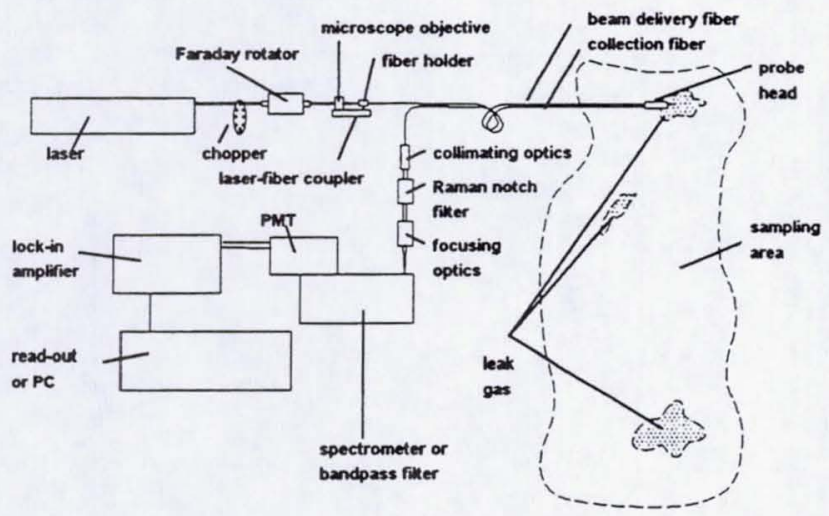

Fig 4: Raman leak detection data acquisition schematic

Because of the chopped laser source, the resulting light signal scattered by the molecules is also a near square wave signal. It is collected by the optical head and transported by means of a 200 $\mu \mathrm{m}$ diameter fiber to a data reduction facility. The $200 \mu \mathrm{m}$ diameter is selected to facilitate alignment and coupling of the collected light inside the probe head. Light emerging from the fiber is collimated with an anti-reflective coated lens. The optical notch filter requires collimated light. This is accomplished by matching the numerical aperture of the collection fiber with the correct $F_{\#}$ lens. An optical Raman notch filter with an optical attenuation of $10^{-6}$ at the laser line filters the Rayleigh light and the stray laser light. Rayleigh scattering is three orders of magnitude stronger than Raman scattering. With the notch filter, interference due to the Rayleigh process is negligible. Transmission of the notch filter near the hydrogen Raman line is $93 \%$ and at the nitrogen Raman line about $90 \%$, retaining nearly all Raman signal. A lens identical to the collimating lens refocuses the collimated beam, after it passes through the notch filter, into a 0.5 meter spectrometer with a 1:1 image to object ratio. This gives an effective $F_{\#}$ of 4.2 of the refocusing optics.

The 0.5 meter spectrometer with a $F_{\#}=4.0$ is scanned over the Raman lines of hydrogen at $654.6 \mathrm{~nm}$ and nitrogen at $584.6 \mathrm{~nm}$. The spectrometer is equipped with a 300 groove $/ \mathrm{mm}$ grating. The resulting dispersion is $6.4 \mathrm{~nm} / \mathrm{mm}$. Because we are not interested in resolving the spectral structure but only want to measure the total scattered Raman intensity over all Q-branch transitions, this dispersion is sufficient. At ambient temperature, the hydrogen Raman lines of the Qbranch with $514.5 \mathrm{~nm}$ laser excitation are spread between $650.6 \mathrm{~nm}$ and $654.6 \mathrm{~nm}$. The entrance slit size of the spectrometer is set to match the fiber diameter of $200 \mu \mathrm{m}$ for maximum signal capture Setting the exit slit size to $700 \mu \mathrm{m}$ with a spectrometer setting of $652.6 \mathrm{~nm}$ assures capture of all these lines within the optical detector. Because of availability, a bialkali PMT with a quantum efficiency of $5 \%$ at $650.0 \mathrm{~nm}$ is used to convert the detected light into a current. A $1 \mathrm{kOhm}$ resistor converted the current into a voltage. Tests were performed with a $200 \mathrm{MHz}$ pre-amplifier as a replacement of the resistor. A ground loop, however, caused interference between the preamplifier and the lock-in amplifier, and the use of a pre-amplifier had to be abandoned.

The PMT signal is input to the lock-in amplifier, combined with the chopper frequency reference, and processed. Two approaches to the signal processing are pursued. In order to verify that the signal is generated by the molecules, spectral scans across the nitrogen and hydrogen Raman lines are performed. A calibration is taken in a vacuum and a subsequent measurement in an environment with a pressure of $0.1 \mathrm{MPa}$ containing the species to be detected as one of the constituents.

For leak detection it is only necessary to study the change in signal at the Raman line. Scanning a section of the spectrum therefore is not needed. The signal strength with background subtracted is a direct measure of the hydrogen concentration. Calibration is done by measuring the signal strength in a vacuum and with a known quantity of the gas at ambient temperature and pressure. 
Since the measured signal is linearly proportional to the concentration, this calibration is sufficient for the extraction of the fraction of leak gas.

\section{Results}

To simulate leak detection, a test was set up in a laboratory. A test vessel, shown in Figure 5 was filled with a premixed mixture of $2.12 \%$ hydrogen in air at ambient pressure and temperature. This is about half the concentration of the ignition limit of hydrogen in air. This concentration was used as a calibration because of the availability of premixed cylinders of this mixture that could be used to fill the vessel. The use of the vessel was needed to contain the hydrogen and to allow the determination of signal strength versus known concentration for calibration purposes. As compared to the planned in-situ measurements, the use of this vessel did introduce additional problems due to scattering from windows and vessel walls. Successful tests, however, virtually assures the potential for in-situ measurements. They will be followed up by field tests.

The optical probe was directed through one of the observation ports mounted in the test vessel. Scattering from the windows caused some extra interference. The probe was therefore positioned at an angle of $6^{\circ}-8^{\circ}$ to the vessel to reduce contributions from the window scattering. Such scattering is not expected during actual measurements where no windows will be present. The laser beam left the vessel through an optical quality window mounted across from the entry window. This reduced scattering inside the vessel by a significant amount..

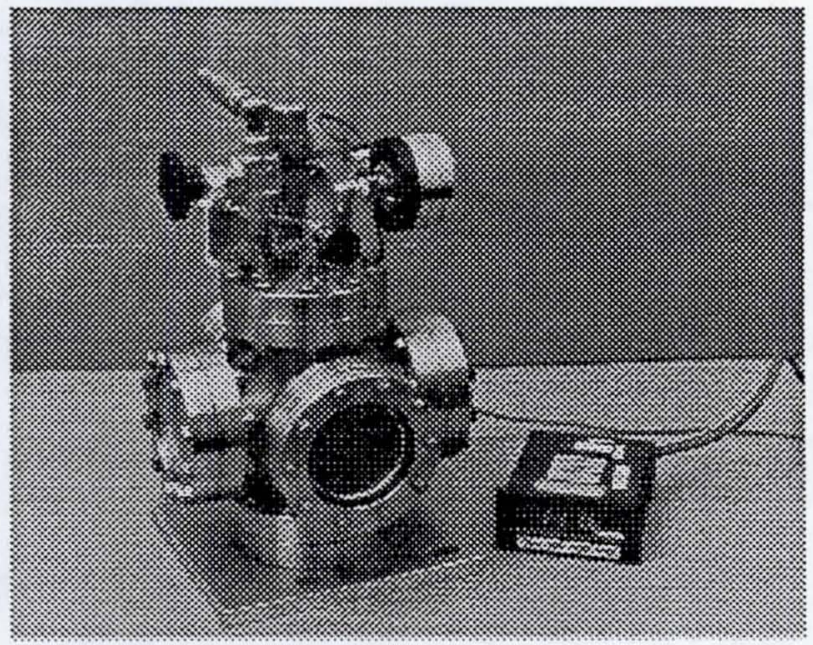

Fig 5: Test vessel containing $2.12 \%$ hydrogen in air
The effect of stray light from scattering surfaces was investigated by evacuating the vessel with a vacuum pump to about $1 \mathrm{~Pa}$ pressure. This caused only stray scattered light from windows and walls to contribute to the signal. The signal at a spectral location of $654.6 \mathrm{~nm}$ was observed with and without laser exposure. A representative trace is shown in Figure 6. The gradual slope after laser turn off/on was due to the temporal response of the detection system. It is clear from the figure that the laser contributed significantly to the background noise, despite all the filters. But because it is a flat background level, a combination of correcting for fluctuating laser output and time running averaging during scanning would eliminate the effect on the signal measurement. For species concentration measurement, the back ground can be calibrated out by generating a calibration curve of species concentration versus intensity. This yields a linear relationship, where the line that represents the relationship indicates the level of the background noise at the zero concentration mark.

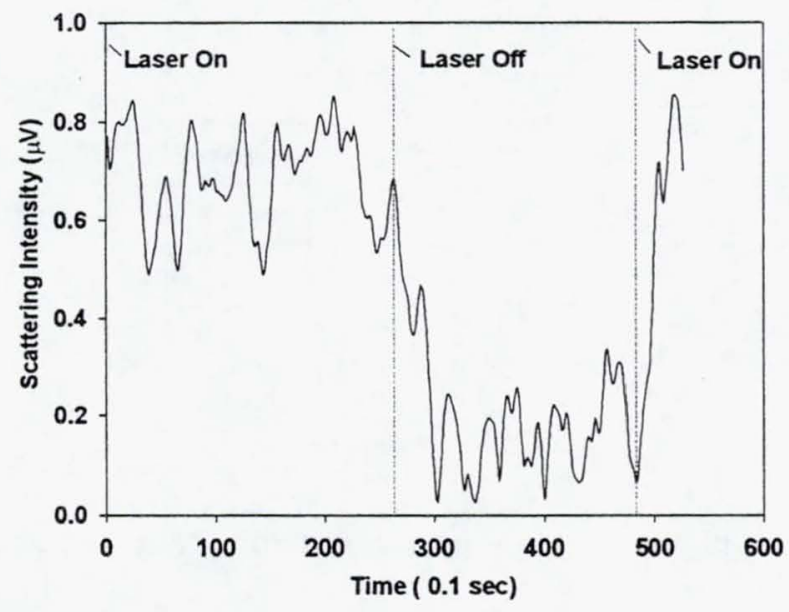

Fig 6: Background scattering due to laser light

A first series of experiments was performed by scanning the spectrometer across the Raman lines of nitrogen and observing the scattered signal with the lock-in detector. Two sets of measurements were taken. One in a well lit room and one in a darkened laboratory. Each scan was repeated three times and shown in Figure 7 . The scans with ambient light run from $577.0 \mathrm{~nm}$ to $590.0 \mathrm{~nm}$ and were taken at $0.1 \mathrm{~nm} / \mathrm{sec}$, giving scan times of two minutes. The lock-in time constant for these scans was set at $1 \mathrm{sec}$. The resulting spectrum was noisy due to the short time constant and ambient light contribution. The scan without ambient light ran from $580.0 \mathrm{~nm}$ to 588.0 $\mathrm{nm}$ with a scan speed of $0.02 \mathrm{~nm} / \mathrm{sec}$, which means that a single scan took 400 seconds to 
complete. The lock-in time constant was 10 seconds. This reduced the noise significantly and greatly improved the signal to noise ratio.

Good nitrogen Q-branch spectra were obtained, for both the faster and slower scans. The Q-branch was broader than the prediction due to the large slit size and the scanning method where the received light was integrated over a finite spectral range, while the spectrum was scanned. It is clear from the figures that the absence of ambient light significantly improved the signal to noise ratio. Chopping the signal did not remove all ambient light, even with the highest roll-off lowpass filters. It is important to investigate the source of this contribution, because the detection method is being developed for outdoors as well as indoors. Since the ambient light contribution is accepted by the lock-in detector, it is expected that it penetrates the system through the chopper. Placing baffles and masks and closing off the space between the laser and chopper might improve the signal to noise ratio with ambient light.

Longer time constants will also improve the signal to noise ratio, as expected. However, speed of detection is an important parameter. A balance has to be found between detection speed, sensitivity, and signal to noise ratio.

The results of three initial attempts to detect hydrogen are shown in Figure 8 . The nitrogen partial pressure inside the vessel was $77 \%$. The hydrogen partial pressure was $2.12 \%$. This low hydrogen concentration resulted in a much weaker signal than the nitrogen scattered signal. The three scans were obtained with a scan speed of 0.02 $\mathrm{nm} / \mathrm{sec}$, from $648.0 \mathrm{~nm}$ to $658.0 \mathrm{~nm}$. The lock-in time constant used to acquire the intensity data was 10 seconds, sufficiently long to average the noise. A small increase of the intensity was seen where the hydrogen Raman line was expected, encouraging the continued development of the diagnostic. In these experiments, the signal barely exceeded the noise level. It was further evident that the signal level varied from scan to scan, such that the noise level in one scan equaled the signal level in the next. This could be caused by mode hopping of the laser, where a change in modal structure on the beam results in a sudden change in transmitted laser power.

\section{Potential Improvement}

Tests were performed with a correction of the signal to account for fluctuating laser power. The laser power was measured by splitting off $4 \%$ of the beam at the laser exit, before the chopper and to divide the signal level by the laser power. This proved to be incorrect. The laser exhibited a large degree of mode hopping, fluctuating in power between 700 and $900 \mathrm{~mW}$. This did not translate however in a proportional level of power delivered at the probe head. This is possibly due to the limited number of modes supported by the fiber, which could be interpreted as being a modal filter. To correctly account for variations in laser power it is necessary to embed a photo detector inside the optical head, where light scattered by the graded index lens provides an acceptable laser intensity measurement. A bandpass filter around the laser line will reject contributions from the environment.
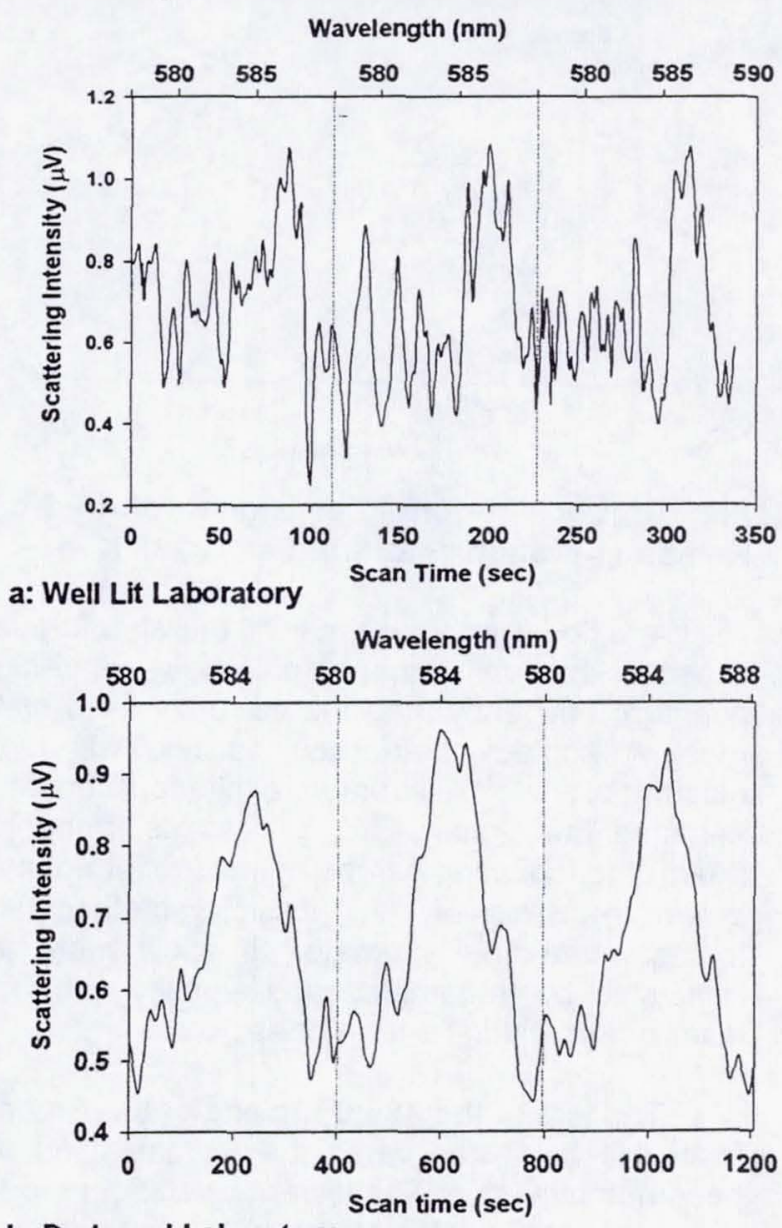

b: Darkened Laboratory

Fig 7: Lock-in detection signal of the nitrogen Q-branch spectra at $T=298 \mathrm{~K}$. a: scan speed 0.1 $\mathrm{nm} / \mathrm{s}$, range $5770-5900 \mathrm{~A}$; b: scan speed 0.02 $\mathrm{nm} / \mathrm{s}$, range $5800-5880 \mathrm{~A}$.

A significant improvement of the signal to noise ratio is expected by a redesign of the optical probe, where replacement of the dichroic mirror by a fully reflective mirror with a hole in the center for laser beam passage will strongly reduce scattering. 
In the current design, two dichroic mirrors are installed in the laser beam to maintain the position of the beam on the optical axis. This gives four scattering surfaces which strongly contribute to the noise level. A reduction of noise contributions due to ambient light is expected by covering the laser beam path from the laser to the fiber. Subsequent background noise reduction is expected by the judicious placement and testing of more baffles, pinholes and optical filters.

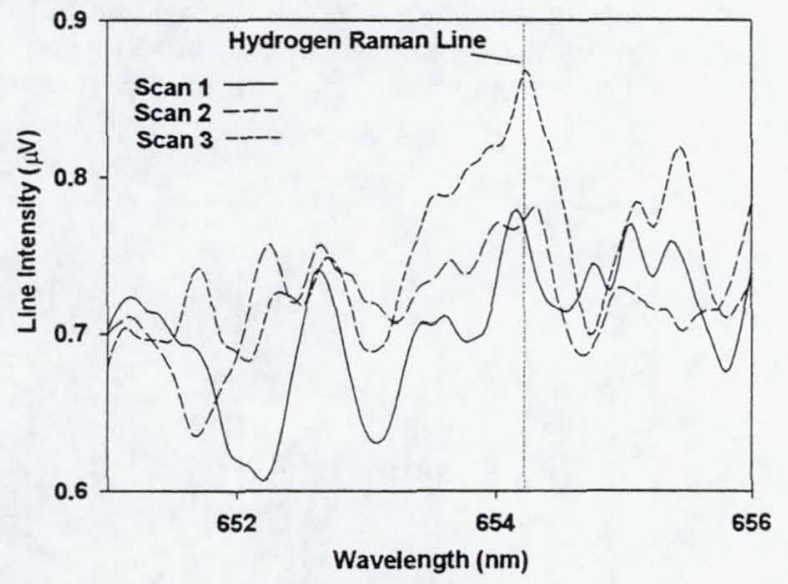

Fig. 8: Lock-in amplifier signal of hydrogen Raman Q-branch scattering at $T=298 \mathrm{~K}$

Additional improvement of the signal level is possible by utilizing photo tubes with higher quantum efficiency than the currently used bialkali tube. A cooled photo tube housing will reduce electronic noise. A stronger excitation source will enhance the signal. This is possible if the laser beam-fiber coupling can be optimized for enhanced power transmission. A possible method is to increase the fiber diameter to allow more laser modes to be transmitted and a laser with higher laser power within fewer modes .

The use of the $488.0 \mathrm{~nm}$ line of the Argon-Ion laser will be tested when the Raman notch filter becomes available. The lower wavelength provides a stronger scattering signal.

\section{Conclusions}

Both nitrogen at high concentrations and hydrogen at low concentrations have been detected with spontaneous Raman scattering technique. A probe has been designed and used to accomplish these measurements in the back scatter direction to facilitate access of difficult to reach locations. Preliminary measurements in a laboratory setting with adverse conditions caused by windows and walls show that high concentrations are easily detected but low concentrations require improvement in sensitivity and signal to noise ratio. In-situ measurements are expected to experience less noise, due to the absence of vessel windows and walls. It is anticipated that they will present alternate problems, however, which will be determined by field testing.

The current tests have shown that species detection and identification with the proposed instrument is possible in an enclosed environment. Future work will focus on changing the data acquisition scheme for verifying the presence of the species without spectral scanning. The transfer from laboratory tests to in-situ measurements is a priority. Further tests have to show the sensitivity and speed that can be obtained, and the possibility of detecting multiple species simultaneously. The potential use of this device in process control is being investigated.

\section{Acknowledgement}

I would like to thank Dave Manzella for his invaluable help in setting up the data acquisition system.

\section{$\underline{\text { References }}$}

${ }^{1}$ Rhodes, R., Personal Communication, KSFC, May 1994.

${ }^{2}$ Adler-Golden, S. M., Goldstein, N., Bien, F., Matthew, M. W., Gersh, M. E., Cheng, W. K., and Adams, F. W., "Laser Raman sensor for measurement of trace-hydrogen gas," Applied Optics, Vol.31, No. 6, February 1992, pp 831-835.

${ }^{3}$ Caputo, B., "Hydrogen Detection Lidar," Computer Genetics Corporation, VEN-1519, Final Report NASA Contract \#NAS10-11421, 1988

${ }^{4}$ Long, D. A., "A partial quantum mechanical treatment of Rayleigh and rotational and vibrational Raman scattering," Raman Spectroscopy , McGrawHill, Great Britain, 1977, pp. 74-111.

${ }^{5}$ Shirley, J. A., "Investigation of the Feasibility of Temperature Profiling Optical Diagnostics in the SSME Fuel Pre-Burner," NASA R83-956181F, June 1983

${ }^{6}$ Asawaroengchai,, C., and Rosenblatt, G. M., "Rotational Raman Intensities and the Measured Change with Internuclear Distance of the Polarizability Anisotropy of $\mathrm{H}_{2}, \mathrm{D}_{2}, \mathrm{~N}_{2}, \mathrm{O}_{2}$, and $\mathrm{CO}$," The Journal of Chemical Physics, Vol. 72, No. 4, February 1980, pp. 2664-2669. 
${ }^{7}$ Murphy, W. F., Holzer, W., and Bernstein, H. J., 'Gas Phase Raman Intensities: A Review of PreLaser Data," Applied Spectroscopy, Vol. 23, 1969, pp. 211-218.

${ }^{8}$ James, T. C. and Klemperer, W., "Line Intensities in the Raman Effect of ${ }^{1} \Sigma$ Diatomic Molecules," The Journal of Chemical Physics, Vol. 31, No. 1, July 1959, pp. 130-134.

9Dicke, R. H., "The Effect of Collisions Upon the Doppler Width of Spectral Lines," Physical Review, Vol. 89, 1953, pp. 472

${ }^{10}$ Duncan, A. B. F.,"Theory of Infrared and Raman Spectra,"Chemical Applications of Spectroscopy,1st ed., 1956, pp 188-245.

${ }^{11}$ Lederman, S.,"The Use of Laser Raman Diagnostics in Flow Fields and Combustion," Progress in Energy Combustion Science, Vol.3, 1977, pp 1-34.

\section{Appendix A: General Raman Scattering Theory}

Molecules inside an electromagnetic field experience perturbations of their internal structure. Energy exchange between the field and the molecules may cause excitation of the molecules. Such molecules return to an equilibrium state by radiating the excess energy absorbed from the EM field. A fraction of the excited molecules return to their initial state while radiating energy equal to the absorbed energy. This is Rayleigh scattering. A small fraction of the excited molecules will decay to a different vibrational and/or rotational energy state. The radiated energy shows up with a frequency slightly shifted from the absorbed frequency. This is Raman scattering and the change in energy is referred to as a Raman shift. The Raman shift is dependent on the energy difference between the vibrational and/or rotational states before and after the perturbation, referred to as the initial and final states. This frequency is species dependent and can be used to identify molecules. In order to describe details of the Raman scattering, different approaches are used. An energy level description is used to find the spectral location of the individual Raman scattered lines. Wave mechanics is used to understand the individual line strengths. Additionally, if a measurement technique relies on the polarization properties of the scattered light and/or the angular dependence of the Raman scattering based on the molecule's polarization is significant, it is necessary to use an electromagnetic description.
Spectral Location

To find the spectral location of Raman emission radiated by molecules exposed to laser radiation, a quantum mechanical description is used. The rotational and vibrational energy of a molecule in equilibrium can be described by the rotational and vibrational frequency and rotational and vibrational quantum numbers, $v$ and $J$. Energy exchange between the molecule and the electromagnetic field brings the internal energy to an unstable higher state by the amount equal to $\left(h v_{0}\right)$ where $h$ is Planck's constant and $v_{0}$ is the frequency of the laser light. The molecule returns to an equilibrium level by emitting a photon with an energy equal to the absorbed energy plus the difference energy between the initial and final state. The location of any Raman line can be given by:

$$
v_{R}=v_{0} \pm\left[\left(E_{v^{\prime} J^{\prime}}-E_{v^{\prime \prime} J^{\prime}}\right) / h\right]
$$

where $v_{R}$ is the frequency of the Raman signal, and $E_{v^{\prime} J^{\prime}}$ and $E_{v^{\prime \prime}} J^{\prime \prime}$ are the rotational-vibrational energy levels of initial and final states, also called the Raman shift. These energy levels are particular to a specific molecule. Each molecule under exposure of laser light with a given frequency therefore emits Raman scattered light at a frequency characteristic for that molecule.

\section{Line Intensity}

When we observe the spectral distribution of radiation emitted by a gas volume exposed to incident laser light, we detect a number of lines. The distribution of signal between these lines depends on the number and type of polyatomic gases. There will always be a strong Rayleigh line at the laser frequency, contributed to by all polyatomic gases. There are also a number of Raman lines shifted from the laser line, each corresponding to a specific gas species. The intensity of each Raman line as observed with a spectroscopic system is given by: ${ }^{11}$

$$
I_{R}=I_{0} C N_{v^{\prime},}, v_{R}^{4}\langle\alpha\rangle_{\pi}^{2}
$$

where $I_{0}$ is the intensity of the incident laser and $\mathrm{N}_{v^{\prime},}$ ' is the number of molecules with an initial energy state that generates this Raman line described by vibrational and rotational quantum number $V^{\prime}$ and $J$ '. $C$ is a constant which includes the solid angle over which the Raman radiation is collected, the efficiency of the collection optics and the spectral response of the detector. Again, $v_{R}$ is the frequency of the Raman line as given by $\mathrm{Eq}$. A.1. 
The term $\langle\alpha\rangle_{i j}{ }^{2}$ is the transition probability or the probability that a molecule within the molecular population $\mathrm{N}_{v, j}$, radiates light under the influence of laser irradiation at the frequency $v_{\mathrm{R}}$. The subscripts $f$ and $i$ indicate the final and initial energy state. In order to calculate this transition probability it is necessary to introduce the molecular polarizability. To understand the molecular polarizability, consider the electrons and nuclei of a homogeneous diatomic molecule in equilibrium, which are normally distributed such that no permanent electric dipole results. When placed in an electromagnetic field, these particles are displaced due to the electric field and form an induced dipole. The strength of this dipole depends on the ease with which such a dipole is formed. This susceptibility of the molecule to the electric field is called the polarizability.

The strength of the induced dipole also depends on the relative orientation of the electric field with respect to the molecular axis. For a homonuclear diatomic molecule, the strength of the dipole moment generated when the electric field is parallel to the internuclear axis is different than the strength of the dipole moment generated when the field is perpendicular to the axis. The two dipole moments perpendicular to the internuclear axis are the same, but for more complex molecules all three axes generally have different polarizabilities.

The induced dipole moment for a molecule with random orientation within the electric field can be found by a vector combination of the three dipole components along the main axis. If the strength of the induced dipole moment for a given orientation is represented by a vector with its origin at the molecular center, the end point of that vector lies on an ellipsoid of which the main axis, in the case of the diatomic molecule, coincide with the main molecular axis. This ellipsoid is the polarizability ellipsoid, a tensor which mathematically can be given in matrix notation by the matrix elements $\alpha_{x x^{\prime}}, \alpha_{y y}, \alpha_{z z}, \alpha_{x y}, \alpha_{x z}$, and $\alpha_{y z}$. The matrix is symmetric around its diagonal.

Incident light has an oscillatory electric field which in turn creates an oscillatory induced dipole moment in the molecule. In addition, the dipole moment varies due to molecular rotation and vibrational motion, which causes the internal potential energy to change. All these factors influence the strength and frequency of the oscillating dipole moment and the resulting radiation.
The intensity of the radiation emitted by an oscillating dipole is proportional to the square of the time averaged dipole moment. Because this dipole moment is linear with the polarizability, the total intensity observed will be proportional to the square of the polarizability tensor matrix. This term is the transition probability, $\langle\alpha\rangle_{f i}{ }^{2}$ which can now be defined in terms of the matrix elements of the polarizability tensor: ${ }^{10}$

$$
<\alpha_{x y}>_{f}=\int \psi_{v^{\prime} J^{\prime}}^{*} \alpha_{x x} \psi_{v^{\prime} J^{*}} d x d y
$$

where $\psi^{*}{ }_{v^{\prime} J^{\prime}}$ and $\psi_{v^{\prime \prime} J^{\prime \prime}}$ are the rotational-vibrational wavefunctions of the initial and final states. The integral is over all coordinate space. Additional elements can be obtained by substitution of $x, y$, and $z$. When the initial and final states are the same, the resulting term gives the transition probability for Rayleigh scattering. Raman scattering results when the initial and final states are different. When the initial vibrational state is lower in energy than the final state, the scattered photons form what is called the Stokes Branch. The anti-Stokes branch is formed when the initial vibrational state has a higher energy than the final state. There is also a terminology for rotational transitions. The O-branch results when the initial rotational state is at higher energy than the final state, the Q-branch results when the initial and final rotational states have the same energy and the Sbranch shows up for transitions from a lower energy to a higher energy rotational state.

James and Klemperer ${ }^{8}$ simplify Eq. (A.3) for the class of ${ }^{1} \Sigma$ diatomic molecules, to which both nitrogen and hydrogen belong, into expressions for the line strengths, expressed in the two components of the polarizability tensor, along and perpendicular to the internuclear axis, $\alpha_{\mu}$ and $\alpha_{\perp}$ respectively. Quantities which are important in intensity calculations are the isotropic and anisotropic parts of the polarizability tensor, which are defined as:

$$
\begin{aligned}
& \alpha=\frac{1}{3}\left(\alpha_{/ /}+2 \alpha_{\perp}\right) \\
& \beta=\alpha_{/ /}-\alpha_{\perp}
\end{aligned}
$$

An additional quantity is defined as:

$$
\chi=\frac{\beta_{\mathrm{e}}}{r_{\mathrm{e}} \beta_{\mathrm{e}}{ }^{\prime}}
$$


where $r_{e}$ is the equilibrium internuclear distance and $\beta_{\theta}$ and $\beta_{\theta}^{\prime}$ are the anisotropy and derived anisotropy.

$$
H(J)=\frac{J\left(4 J^{2}+1\right)}{(2 J+1)(2 J-1)}
$$

Shirley ${ }^{5}$ uses these results to evaluate Raman intensities for the hydrogen spectra for temperatures from $600 \mathrm{~K}$ to $1200 \mathrm{~K}$. He expresses the polarizability matrix elements derived by James and Klemperer as:

$$
<\alpha\rangle^{2}=C_{b} S(J) f(J)
$$

where $C_{b}$ is an intensity factor which depends on the rigid rotator polarizability, $S(J)$ is the rotational line strength, and $f(J)$ is a correction for the centrifugal distortion caused by vibrationalrotational interaction.

Rotational-vibrational correction factors are included in Shirley's work and are given here for the rotational-vibrational Q-branch only. The three intensity factors and corresponding line strengths are calculated for observation in the back scatter direction. Significant correction factors due to the centrifugal distortion are reported for rotational levels of $\mathrm{J}=5$ and 6 . However, for the ambient temperature applications, where only levels up to $\mathrm{J}=3$ are significantly populated, this correction factor is negligible.

The Q-branch intensity in the back scatter direction including correction factors are:

$$
\begin{aligned}
I_{01}^{Q}= & \left(2 \pi \frac{v_{R}}{C}\right)^{4}\left(\frac{B_{\theta}}{\omega_{\theta}}\right) f_{01}^{Q}(J) N_{O J} \\
& x\left[\left(r_{\theta} \alpha_{\theta}^{\prime}\right)^{2}+S(J)\left(r_{\theta} \gamma^{\prime}\right)^{2} /(2 J+1)\right]
\end{aligned}
$$

where $v_{R}$ is the Raman shift, $\mathrm{c}$ is the speed of light, and $B_{e}$ and $\omega_{e}$ are rotational and vibrational constants respectively. $N_{0,}$ is the population of the initial rotational vibrational energy state with $v^{\prime}=0$, $\alpha_{e}{ }^{\prime}$ and $\gamma^{\prime}$ (or $\beta_{e}$ ) are the first derivatives of the isotropic and anisotropic parts of the polarizability tensor at equilibrium, and $\mathrm{J}$ is the rotational quantum number. The correction for the centrifugal distortion is given by:

$$
f_{01}^{Q}(J)=\left[1-3\left(\frac{B_{0}}{\omega_{0}}\right)^{2} J(J+1)\right]^{2}
$$

and the line strength by:

$$
\begin{aligned}
S(J)= & \frac{1}{15} \frac{J(J+1)(2 J+1)}{(2 J+3)(2 J-1)}-\frac{2 J+1}{9}+\frac{4}{15} \frac{J(J+1)}{2 J+1} \\
& +\frac{H(J)+H(J+1)}{15}
\end{aligned}
$$

with 
Public reporting burden for this collection of information is estimated to average 1 hour per response, including the time for reviewing instructions. searching existing data-sources. gathering and maintaining the data needed, and completing and reviewing the collection of information. Send comments regarding this burden estimate or any other aspect of this Davis Highway, Suite 1204. Arlington, VA $22202-4302$, and to the OHtice of Management and Budget, Paperwork Reduction Project (0704-0188). Washington. DC 20503.

\begin{tabular}{|l|l|r|}
\hline 1. AGENCY USE ONLY (Leave blank) & $\begin{array}{r}\text { 2. REPORT DATE } \\
\text { August } 1994\end{array}$ & $\begin{array}{r}\text { 3. REPORT TYPE AND DATES COVERED } \\
\text { Final Contractor Report }\end{array}$ \\
\hline
\end{tabular}

4. TITLE AND SUBTITLE

The Use of Spontaneous Raman Scattering for Hydrogen Leak Detection

Wim A. de Groot

7. PERFORMING ORGANIZATION NAME(S) AND ADDRESS(ES)

NYMA, Inc.

2001 Aerospace Parkway

Brook Park, Ohio 44142
5. FUNDING NUMBERS

$$
\text { WU-506-42-31 }
$$

C-NAS3-25266
9. SPONSORING/MONITORING AGENCY NAME(S) AND ADDRESS(ES)

National Aeronautics and Space Administration

Lewis Research Center

Cleveland, Ohio 44135-3191
8. PERFORMING ORGANIZATION REPORT NUMBER

E-9057

11. SUPPLEMENTARY NOTES

Prepared for the 30th Joint Propulsion Conference cosponsored by AIAA, ASME, SAE, and ASEE, Indianapolis, Indiana, June 27-29, 1994. Research done when Wim A. de Groot was with Sverdrup Technology, Inc., 2001 Aerospace Parkway, Brook Park, Ohio 44142. Project Manager, Steven S. Schneider, Space Propulsion Technology Division, NASA Lewis Research Center, organization code 5330, (216) 433-7484.

12a. DISTRIBUTIONAVAILABILITY STATEMENT

Unclassified -Unlimited

Subject Categories 72 and 20

10. SPONSORING/MONITORING AGENCY REPORT NUMBER

NASA CR-195373

AIAA-94-2983

13. ABSTRACT (Maximum 200 words)

A fiber optic probe has been built and demonstrated that utilizes back scattered spontaneous Raman spectroscopy to detect and identify gaseous species. The small probe, coupled to the laser and data acquisition equipment with optical fibers, has applications in gaseous leak detection and process monitoring. The probe design and data acquisition system are described. Raman scattering theory has been reviewed and the results of intensity calculations of hydrogen and nitrogen Raman scattering are given. Because the device is in its developmental stage, only preliminary experimental results are presented here. Intensity scans across the rotational-vibrational Raman lines of nitrogen and hydrogen are presented. Nitrogen at a partial pressure of $0.077 \mathrm{MPa}$ was detected. Hydrogen at a partial pressure of $2 \mathrm{kPa}$ approached the lower limit of detectability with the present apparatus. Potential instrument improvements that would allow more sensitive and rapid hydrogen detection are identified.

\section{SUBJECT TERMS}

Raman scattering; Leak detection; Hydrogen

16. PRICE CODE

$\mathrm{AO3}$

\begin{tabular}{|c|c|}
\hline $\begin{array}{c}\text { 17. SECURTY CLASSIFICATION } \\
\text { OF REPORT }\end{array}$ & $\begin{array}{c}\text { 18. SECURTY CLASSIFICATION } \\
\text { OF THIS PAGE } \\
\text { Unclassified }\end{array}$ \\
\begin{tabular}{c} 
Unclassified \\
\hline
\end{tabular}
\end{tabular}

19. SECURITY CLASSIFICATION OF ABSTRACT Unclassified

\section{LIMITATION OF ABSTRACT}

